\title{
The Isolated Gamma Probe Technique for Sentinel Node Penile Carcinoma Detection Is Unreliable
}

\author{
Lucio F. Gonzaga-Silva, Jose M. Tavares, Fernando C. Freitas, Manoel E. Tomas Filho, \\ Vladmir P. Oliveira, Marcos V. Lima
}

Department of Surgery, Federal University of Ceara and Section of Urology, Cancer Hospital of Ceara, Fortaleza, Ceara, Brazil

\begin{abstract}
Purpose: Penile carcinoma is a common disease in northeast Brazil. This paper shows the results of the use of isolated gamma probe and discusses the incidence of false negative rates.

Materials and Methods: From July 2000 to September 2003, 27 newly diagnosed penile carcinoma patients (T1, T2, N0) were included in this prospective study. The isolated gamma probe technique uses the sodium phytate technetium as a tracer and inguinal scanning with probe and after identified the lymph node it is removed. Lymphadenectomies were performed for positive inguinal lymph nodes metastasis.

Results: There were 27 patients (mean age 59.6). Follow up was 37 months. Patients from country were $72 \%$ and illiterate or semi-illiterate were $56.7 \%$. The tumors were mostly located in the glans $(81.4 \%)$. They were T1, 52 \% and T2, $48 \%$. $81.4 \%$ of the patients underwent partial penectomy, and $18.6 \%$ underwent postectomy and excision with wide margins. In $48 \%$ of the patients, the highest radioactive count rate was located on the left side, while in $41 \%$ was located on the right side. Only one patient had a positive pathological lymph node metastasis at the moment of the surgery. Additionally 3 patients became inguinal lymph node positive at the follow up. This date yielded a sensibility rate of $25 \%$ and a falsenegative rate of $42.8 \%$.

Conclusion: Isolated gamma probe technique for sentinel node penile carcinoma has a very low sensibility and a high false negative rate. Therefore it is highly advisable the addition of others methods such as lymphoscintigraphy, vital blue, ultrasonography and so on. The isolated gamma probe technique for sentinel node penile carcinoma detection is unreliable.
\end{abstract}

Key words: penile cancer; lymphatic metastasis; sentinel lymph node biopsy; gamma probe technique Int Braz J Urol. 2007; 33: 58-67

\section{INTRODUCTION}

Though rare in developed countries, penile carcinoma is relatively common in Latin America (1). On the average, 3 new cases have been identified at our Hospital every month over the last 5 years (2).
The lymphatic system is the primary route for metastasis. Tumors spread loco-regionally and stepwise (3). Survival of patients with penile carcinoma clearly depends on the status of the inguinal lymph node (4). Thus, the overall 5-year survival rate is $73-95 \%$ for patients with negative inguinal lymph 
nodes and $19-62 \%$ for patients with positive inguinal lymph nodes (5). When associated with pelvic disease, the 5-year survival rate is less than $10 \%$. If left untreated, patients with metastasis rarely survive for longer than 2 years (6).

In patients with metastasis confined to the regional lymph nodes, inguinal lymphadenectomy is very often curative, with disease-free rates of 30-90\% (7). Inguinal lymphadenectomy has been associated, in some reports, with significant levels of morbidity $(30-50 \%)$ and up to $3 \%$ of mortality $(8,4)$. Moreover, prophylactic bilateral inguinal lymph node dissection is considered unnecessary in up to $80 \%$ of penile carcinoma patients with clinically negative regional lymph nodes (9).

Elective bilateral inguinal lymph node dissection is the most invasive approach and the least invasive is a "wait and see" policy (10). However, the latter is associated with a poorer prognosis if tumor-involved lymph nodes become palpable during follow-up $(11,12)$.

Dynamic sentinel node biopsy provides a means of assessing lymph node status in the management of penile carcinoma, through a minimally invasive procedure. It has important diagnostic, prognostic and therapeutic value at the cost of only minor morbidity and is an attempt to diminish the risk of complications without jeopardizing the oncological results (10).

The gamma probe technique has been adopted at our institution since July 1999. However, it was used alone (without lymphoscintigraphy and vital dye) on the first 27 patients mainly because our hospital had not yet been officially authorized to use the gamma camera.

After the use of the equipment had been authorized (September 2003), the complete approach (gamma probe, lymphoscintigraphy and vital blue dye) became the routine procedure in the investigation of penile carcinoma sentinel nodes.

The purpose of this paper was to show the results of the isolated use of gamma probe technique for sentinel node investigation in patients with penile carcinoma at our institution, before the complete technique was introduced in September 2003, and to analyze its value as a less invasive lymphatic staging method.

\section{MATERIALS AND METHODS}

Between July 2000 and September 2003, 27 patients with newly diagnosed penile squamous cell carcinoma were prospectively enrolled for sentinel node detection by gamma probe technique. Informed consent was obtained from all patients and the protocol was approved by the research ethics committee at our institution.

The 27 patients enrolled in the study did not differ significantly from subjects described in similar studies published in the literature. The mean age was 59.6 years (range 41-80). The Mean follow-up time was 37 months (range: 24-62). Five patients were lost to follow up (Table-1).

Patients were eligible when staged T1-T2, N0, according to the 2002 TNM classification system of the International Union Against Cancer.

At our hospital, technetium-99-m-labeled sodium phytate ( $99 \mathrm{mtc}$ ) is used in colloidal presentation as a tracer. A dose of $0.2 \mathrm{~mL}$ was injected subdermally at the four cardinal points around the penile lesion, totaling $0.8 \mathrm{~mL}$. The average radioactive dose was (59 MBq) (13).

The bilateral inguinal probe scanning was performed about 30 minutes after the injection. First, background count is established by measuring radioactivity over a neutral site. Then the handheld gamma detection probe was used to identify the sentinel node sites. A sentinel node was defined as a node with radioactivity three times over the background.

Finally, the focus of activity in the inguinal area (the so-called hot point) was marked off. A small incision was then made and the sentinel node was removed. When sentinel lymph nodes are removed successfully, the radioactivity count rate on the excision site should not exceed $10 \%$ of the highest exvivo radioactivity count.

The excised sentinel lymph node was sent to the pathologist after making sure it was the one (on either side) with the highest background radioactivity count (corresponding to the highest quantity of radio colloid).

The sentinel node was bisected, fixed in formalin, embedded in paraffin and sectioned at $8 \mathrm{lev}$ - 
els, on the average (5- $\mu \mathrm{m}$ sections). Paraffin sections were stained with hematoxylin and eosin (13).

Inguinal lymphadenectomies were performed immediately for all inguinal lymph node metastasis proving positive. Negative cases, (i.e. without lymph nodes metastasis) were followed up with check-ups every three months for three years. Patients subjected to inguinal lymphadenectomy were kept under careful observation to identify early or late complications.

Disease-specific survival is defined as the percentage of people in a study who have survived a particular disease (in this case, penile cancer) since diagnosis or treatment. Disease-free survival is the length of time after treatment during which no disease (in this case nodal or distant recurrence) is found.

\section{RESULTS}

Most of the patients were illiterate or semiilliterate $(56.7 \%)$ (From country $72 \%$ ). The tumors were mostly located in the glans $(81.4 \%)$ and in the prepuce $(38 \%)$. Some tumors affected the glans and prepuce $(18.5 \%)$.

As to the tumor stage, $51.9 \%$ were $\mathrm{T} 1$ and $48.1 \%$ were $\mathrm{T} 2$. With regard to treatment, $81.4 \%$ of the patients underwent to partial penectomy, while the remainder had postectomy and excision with wide margins $(18.6 \%)$. In eleven patients $(40.7 \%)$, the highest radioactive count was located only on the left side; in nine patients (33.3\%), it was located only on the right. The radioactive count was too low for sentinel node identification in three patients (11.1\%) (Table-1).

No complications related to the isolated gamma probe technique for sentinel node penile carcinoma detection were observed.

Only one patient exhibited lymph node metastasis at the time of the surgery. The patient was given a bilateral inguinal lymphadenectomy during the same session. The pathological examination of the specimen revealed that the excised sentinel lymph node was the only node affected (Table-1).

In addition, three patients who were negative for pathological lymph node metastasis at the moment of the surgery became inguinal lymph node- positive in the course of follow up. Thus, the sensitivity rate was $25 \%$ and the false-negative rate of $42.8 \%$ (Table-1).

Of the four patients submitted to bilateral inguinal lymphadenectomy three are disease-free at the time of writing (September 2005) and one died of cancer.

\section{COMMENTS}

Prophylactic bilateral inguinal lymph node dissection is considered unnecessary in up to $80 \%$ of penile carcinoma patients with clinically negative regional lymph nodes. The challenger lies in identifying the remaining $20 \%$ of occult metastasis and thereby offers such patients an opportunity for cure (9).

The isolated use of familiar predictive prognostic factors for the diagnosis of occult lymph node metastasis, such as depth of invasion, differentiation grade, and vascular invasion, results in considerable false-positive and false-negative rates (10).

On the other hand, dynamic sentinel node biopsy has proved very useful in the detection of occult lymph node metastasis. The procedure is minimally invasive, preventing unnecessary lymph node dissections, and moreover, improves substantially the staging with a morbidity rate of only $8 \%$ $(10,14)$.

Although dynamic sentinel node biopsy for penile carcinoma was adopted at our hospital in July 2000 , the procedure was restricted to the intra-operative gamma ray technique. Twenty seven patients were studied by this method until September 2003 when the complete technique (including preoperative lymphoscintigraphy, intra-operative gamma ray detection and a vital blue dye) became available.

Sentinel lymph node biopsy is best performed by a committed team with experience in surgery, pathology and nuclear medicine. The nuclear physician is responsible for preparing and measuring the radioactive material and for controlling the measuring and mapping equipment. The surgeon is responsible for handling the probe during the surgery, for the surgical procedures and for the management of the case during follow-up (15). 
Table 1 - Patients age, overall findings, treatment and follow up.

\begin{tabular}{|c|c|c|c|c|c|c|}
\hline Patient (age) & Probe & Histology & Staging & Treatment & DS & Follow up \\
\hline $1(74)$ & (+) L (uni) & $(-)$ & $\mathrm{T} 2$ & PP & DF & $62 \mathrm{~m}$ \\
\hline $2(61)$ & (+) L (uni) & $(-)$ & $\mathrm{T} 2$ & PP & DF & $53 \mathrm{~m}$ \\
\hline $3(49)$ & (+) R (uni) & $(-)$ & $\mathrm{T} 2$ & PP & DF & $3 \mathrm{~m}^{\mathrm{A}}$ \\
\hline $4(49)$ & $(-)$ & & $\mathrm{T} 1$ & Excision & DF & $53 \mathrm{~m}$ \\
\hline $5(76)$ & (+) R (uni) & $(+)$ & $\mathrm{T} 1$ & PP / IBL & DF & $52 \mathrm{~m}$ \\
\hline $6(46)$ & (+) B (bil) & $(+)$ & $\mathrm{T} 2$ & $\mathrm{Em} / \mathrm{IBL}$ & DF & $52 \mathrm{~m}$ \\
\hline $7(63)$ & (+) B (bil) & $(+)$ & $\mathrm{T} 1$ & PP / IBL & DF & $15 \mathrm{~m}^{\mathrm{B}}$ \\
\hline $8(66)$ & (+) R (uni) & $(-)$ & $\mathrm{T} 2$ & PP & DF & $50 \mathrm{~m}$ \\
\hline $9(52)$ & (+) L (uni) & $(-)$ & $\mathrm{T} 1$ & PP & DF & $48 \mathrm{~m}$ \\
\hline $10(69)$ & (+) B (bil) & $(-)$ & $\mathrm{T} 2$ & PP & DF & $48 \mathrm{~m}$ \\
\hline $11(54)$ & (+) L (uni) & $(-)$ & $\mathrm{T} 1$ & Excision & DF & $39 \mathrm{~m}$ \\
\hline $12(41)$ & (+) L (uni) & $(-)$ & $\mathrm{T} 2$ & PP & DF & $06 \mathrm{~m}^{\mathrm{C}}$ \\
\hline $13(55)$ & (+) L (uni) & $(-)$ & $\mathrm{T} 2$ & PP & DF & $40 \mathrm{~m}$ \\
\hline $14(63)$ & (+) B (bil) & $(-)$ & $\mathrm{T} 1$ & $\mathrm{PP}$ & DF & $39 \mathrm{~m}$ \\
\hline $15(76)$ & (+) L (uni) & $(-)$ & $\mathrm{T} 2$ & PP & DF & $22 \mathrm{~m}^{\mathrm{D}}$ \\
\hline $16(80)$ & (+) R (uni) & $(-)$ & $\mathrm{T} 1$ & $\mathrm{PP}$ & DF & $37 \mathrm{~m}$ \\
\hline $17(64)$ & (+) L (uni) & $(-)$ & $\mathrm{T} 2$ & PP & DF & $33 \mathrm{~m}$ \\
\hline $18(42)$ & (+) L (uni) & $(-)$ & $\mathrm{T} 1$ & $\mathrm{P}$ & DF & $33 \mathrm{~m}$ \\
\hline $19(46)$ & $(-)$ & & $\mathrm{T} 1$ & PP & DF & $31 \mathrm{~m}$ \\
\hline $20(60)$ & (+) R (uni) & $(-)$ & $\mathrm{T} 2$ & PP / IBL & Ca Death & \\
\hline $21(60)$ & (+) L (uni) & $(-)$ & $\mathrm{T} 1$ & PP & $\mathrm{DF}$ & $31 \mathrm{~m}$ \\
\hline $22(63)$ & (+) R (uni) & $(-)$ & $\mathrm{T} 1$ & PP & DF & $30 \mathrm{~m}$ \\
\hline $23(50)$ & (+) R (uni) & $(-)$ & $\mathrm{T} 1$ & Mohs surgery & DF & $30 \mathrm{~m}$ \\
\hline $24(74)$ & (+) R (uni) & $(-)$ & $\mathrm{T} 2$ & $\mathrm{PP}$ & AD Death & \\
\hline $25(73)$ & $(-)$ & & $\mathrm{T} 2$ & PP & DF & $1 \mathrm{~m}^{\mathrm{E}}$ \\
\hline $26(74)$ & (+) L (uni) & $(-)$ & $\mathrm{T} 1$ & PP & DF & $26 \mathrm{~m}$ \\
\hline $27(72)$ & (+) R (uni) & $(-)$ & $\mathrm{T} 1$ & PP & DF & $24 \mathrm{~m}$ \\
\hline
\end{tabular}

$N=27$ patients; probe $(+)=$ background count positive; probe $(-)=$ background count negative; $D S=$ disease status; $D F=$ disease free; Ca death $=$ Cancer death, $A D$ Death $=$ death for another disease; $L=$ left side; $R=$ right side $B=$ both sides; uni $=$ unilateral $;$ bil $=$ bilateral $; P P=$ partial penectomy; $P=$ postectomy; $E m=$ emasculation $; I B L=$ inguinal bilateral lymphadenectomy; $m=$ months; $A=$ last follow up = May/2001; $B=$ last follow up $=$ Sep/2002; $C=$ last follow up $=$ Nov/2002; $D=$ last follow up $=$ Feb/2003; $E=$ last follow up Oct/2003. Patients 6,7 and 20 developed inguinal tumor during the follow up $(7,8$ and 6 months).

The overall findings, treatment and follow-up of these initial 27 patients (examined with intraoperative gamma probe only) are illustrated in the Table- 1 .

Unlike the studies by Horenblas (14), which excluded patients with $\mathrm{T} 1$ tumors in view of the low risk of occult metastasis, our study was designed so as to include patients with penile carcinoma staged both T1 and T2 N0, considering the local incidence of late recurrences in such patients (T1).
The Table- 1 shows that in all but three patients the sentinel nodes were visualized by the intraoperative gamma probe technique. At this point, no measures were taken for patients with radioactive count below the background count rate (3 patients). However, as recommended by Horenblas and coworkers, it is now considered mandatory to examine such patients for tumor deposits blocking the passage of tracer (5). 
The vast majority of our patients underwent to partial penectomy, while three patients with very small tumors $(4,11$ and 23$)$ were given conservative surgery. Our research team soon is publishing a description of this approach and its indications.

Only one of $27 \mathrm{~T} 1$ and $\mathrm{T} 2$ patients had a positive histology test (5). He was submitted to inguinal bilateral lymphadenectomy shortly after penectomy, and was alive and disease-free after a follow-up of 36 months.

The Table- 1 shows the number of positive histology tests observed during the total follow-up period (July 2000 to September 2003). The histological positivity was observed in four patients. Thus, the sensitivity of the isolated intraoperative gamma probe technique was $25 \%$.

Inguinal tumor outgrowth after excision of a classified tumor-negative sentinel node or non-visualization is classified as false-negative result (10). The Table- 1 show that three patients presented these features i.e. three patients (6,7 and 20) developed inguinal tumor after a negative sentinel node biopsy.

The false-negative rate is defined as the number of false-negative results divided by the total of positive results plus the false-negative results (10). Our three false-negative results occurred clustered around the beginning of the study and resulted in a false-negative rate of $42.8 \%$.

A false-negative rate of this order clearly indicates that the isolated gamma probe technique is not a reliable way of detecting sentinel nodes in penile carcinoma.

The Netherlands Institute of Cancer (Horenblas et al.) found an initial false-negative rate of $18 \%$ ( 6 of 34 cases). The technique revealed metastasis in 28 of 123 patients and was false-negative in 6 patients (13).

In 2001 important adjustments were made to the procedure of dynamic sentinel node biopsy in penile carcinoma patients at the Netherlands Institute of Cancer (Pathological analysis by serial sectioning and immunohistochemical staining, preoperative ultrasonography with fine-needle biopsy aspiration cytology and preoperative lymphoscintigraphy besides exploration of nonvisualized groin) leading to eradication of false-negative results.
In a study of $70 \mathrm{~T} 2-\mathrm{T} 3$ patients submitted to dynamic sentinel node biopsy (pre-operative lymphoscintigraphy, intra-operative gamma probe and vital blue dye), Perdoná and coworkers (2005) found a false negative rate of $11 \%$ and a sensitivity of $90 \%$ (16).

At our Hospital we had also made adjustments to the procedure (as of September 2003), which now includes preoperative lymphoscintigraphy and intra-operative injection of vital blue dye besides gamma ray detection. These improvements are expected to reduce false-negative rates.

Several studies are presently conducted on a variety of tumors and sites. These studies will no doubt, cautiously endorse sentinel lymph node biopsy. However, sentinel lymph node biopsy can be difficult to master. Most surgeons will agree that it takes considerable experience to correctly identify sentinel lymph nodes; in fact, the Oncology Group of the American College of Surgeons recommends that physicians perform at least 30 sentinel lymph node biopsies as part of their training (17).

\section{CONCLUSION}

Our findings show that the isolated gamma probe technique for sentinel node penile carcinoma is associated with very low sensitivity $(25 \%)$ and high false-negative rates $(42.8 \%)$. The inclusion of other techniques, such as lymphoscintigraphy, vital blue, ultrasonography, is therefore highly advisable.

\section{CONFLICT OF INTEREST}

None declared.

\section{REFERENCES}

1. Donald F: Tumors of the Penis. In: Campbell's Urology, 8th edition. Philadelphia, WB Saunders. 2002; pp. 2945.

2. Gonzaga-Silva LF: Histopathology - Biopsy and Prognostic Factors. In: Practical Guide of Urology, 
Rio de Janeiro, Segmento. 2003; pp. 561-2. [in Portuguese]

3. Tabatabaei S, Harisinghani M, McDougal WS: Regional lymph node staging using lymphotropic nanoparticle enhanced magnetic resonance imaging with ferumoxtran-10 in patients with penile cancer. J Urol. 2005; 174: 923-7; discussion 927.

4. Ornellas AA, Seixas AL, Marota A, Wisnescky A, Campos F, de Moraes JR: Surgical treatment of invasive squamous cell carcinoma of the penis: retrospective analysis of 350 cases. J Urol. 1994; 151: 1244-9.

5. Srinivas V, Morse MJ, Herr HW, Sogani PC, Whitmore WF Jr: Penile cancer: relation of extent of nodal metastasis to survival. J Urol. 1987; 137: 880-2.

6. Ravi R: Correlation between the extent of nodal involvement and survival following groin dissection for carcinoma of the penis. Br J Urol. 1993; 72: 817-9.

7. Kossow JH, Hotchkiss RS, Morales PA: Carcinoma of penis treated surgically. Analysis of 100 cases. Urology. 1973; 2: 169-72.

8. Akduman B, Fleshner NE, Ehrlich L, Klotz L: Early experience in intermediate-risk penile cancer with sentinel node identification using the gamma probe. Urology. 2001; 58: 65-8.

9. Abi-Aad AS, deKernion JB: Controversies in ilioinguinal lymphadenectomy for cancer of the penis. Urol Clin North Am. 1992; 19: 319-24.

10. Kroon BK, Horenblas S, Meinhardt W, van der Poel HG, Bex A, van Tinteren H, et al.: Dynamic sentinel node biopsy in penile carcinoma: evaluation of 10 years experience. Eur Urol. 2005; 47: 601-6; discussion 606.
11. Theodorescu D, Russo P, Zhang ZF, Morash C, Fair WR: Outcomes of initial surveillance of invasive squamous cell carcinoma of the penis and negative nodes. J Urol. 1996; 155: 1626-31.

12. McDougal WS: Carcinoma of the penis: improved survival by early regional lymphadenectomy based on the histological grade and depth of invasion of the primary lesion. J Urol. 1995; 154: 1364-6.

13. Kroon BK, Horenblas S, Estourgie SH, Lont AP, Valdes Olmos RA, Nieweg OE: How to avoid false-negative dynamic sentinel node procedures in penile carcinoma. J Urol. 2004; 171: 2191-4.

14. Horenblas S, Jansen L, Meinhardt W, Hoefnagel CA, de Jong D, Nieweg OE: Detection of occult metastasis in squamous cell carcinoma of the penis using a dynamic sentinel node procedure. J Urol. 2000; 163: 100-4.

15. Lima MV, Tavares JM, Silveira RA, Tomas Filho ME, Silva FA, Silva LF: Inintraoperative use of gamma probe for identification of sentinel node in penile carcinoma. Int Braz J Urol. 2002; 28: 123-9.

16. Perdona S, Autorino R, De Sio M, Di Lorenzo G, Gallo L, Damiano R, et al.: Dynamic sentinel node biopsy in clinically node-negative penile cancer versus radical inguinal lymphadenectomy: a comparative study. Urology. 2005; 66: 1282-6.

17. Cantin J, Scarth H, Levine M, Hugi M, Steering Committee on Clinical Practice Guidelines for the Care and Treatment of Breast Cancer: Clinical practice guidelines for the care and treatment of breast cancer: 13 . Sentinel lymph node biopsy. CMAJ. 2001; 165: 16673. Erratum in: CMAJ 2001; 165: 744.

Accepted after revision:

October 25, 2006

\section{Correspondence address:}

Dr. Lucio Flavio Gonzaga Silva

Rua Dr. José Lino, 141 / 1002, Varjota

Fortaleza, CE, 60165-270, Brazil

E-mail: gonzagalf@ secrel.com.br 


\section{EDITORIAL COMMENT}

In 1994, we started with sentinel node biopsy for penile cancer patients. While acknowledging the pioneering work of Cabanas we deliberately labeled our procedure as a dynamic sentinel node procedure to underscore the static nature of the original description and the dynamic nature of the new one. The dynamism is readily seen on the lymphoscintigrams, giving an almost real-time picture of the individual drainage pattern. Too many individual variations led to false negative findings, explaining the lack of enthusiasm of the original description of the procedure.

There has been a long controversy between proponents of an early lymph node dissection and proponents of a wait and see strategy for clinically node negative patients. An analysis from our institute showed an increased survival for patients who underwent an early lymph node dissection based on tumor positive sentinel node finings. In these series no unnecessary lymph node dissections were done. All patients harbored pathologically proven clinically occult metastases. Lymph node dissection was done at the earliest possible moment (1).

The main problem remained to improve the accuracy of the sentinel node biopsy. Accurate sentinel node biopsies rely on collaboration of nuclear medicine physicians, surgeons and pathologists. Moreover, it relies on the use of all possible methods to exclude false negative findings. In our practice this means preoperative ultrasound with or without fine needle aspiration biopsy, a preoperative lymphoscintigram, marking of the sentinel nodes on the skin, discussing the lymphoscintigram with the nuclear medicine physician, injecting patent blue around the tumor, using a gamma detector and having a protocol for measurements before removal and after removal, palpation of the wound after removal and a strict pathology protocol. With all these refinements, we were able to bring down the false negative rate of the initial series of $22 \%$ to an acceptable $4.8 \%$ (in press European Urology). In our hands sentinel node biopsy for penis cancer has evolved as an important clinical staging tool just as reliable as in melanoma and breast cancer patients. One should realize however, that the procedure was initiated in a specialized cancer center together with surgical oncologists, who pioneered the procedure for breast cancer and melanoma.

Another aspect not often cited is the small size of the Netherlands, making regular outpatientclinic visits easy, follow-up very reliable, with a $100 \%$ literacy within the population and the increasing tendency to centralize management of penile cancer patients. All these aspects have been helpful in developing, analyzing and improving the dynamic sentinel node biopsy.

How different is the situation in Brazil and many other countries. The procedure should be viewed against the above-mentioned elements. A rational choice should be made between the introduction of a sentinel node biopsy program, with all the logistics around it or a straightforward lymph node dissection in a patient without any follow up after initial surgery.

What is clear from this paper is that introducing only some aspects of the procedure is not benefiting the patient and should not be done.

\section{REFERENCE}

1. Ravi R: Correlation between the extent of nodal involvement and survival following groin dissection for carcinoma of the penis. Br J Urol. 1993; 72: 8179. 


\section{EDITORIAL COMMENT}

Defining the presence (or absence) of microscopic metastases within the inguinal lymph nodes in patients with squamous penile cancer and no clinical lymphadenopathy remains a challenge. Selective lymphadenectomy based upon the stage, grade or the presence of vascular invasion within the primary tumor decreases the incidence of unnecessary lymphadenectomy but is clearly imprecise. Based upon the evolving experience initially published by colleagues at the Netherlands Cancer Institute (ref. 10, 13, 14 in the article) Dynamic Sentinel Lymph Node Biopsy with preoperative lymphoscintigraphy with subsequent intraoperative detection of emitted gamma radioactivity using a hand held probe has been shown to be a minimally morbid procedure.

In the current study, the authors studied a cohort of patients using only intraoperative detection, as preoperative lymphoscintigraphy was unavailable. Four patients were eventually found to exhibit lymph node metastasis but only one of four was discovered at surgery leading to a sensitivity of only $25 \%$. One of the three died due to cancer, one was lost to followup at 15 months, and the other is alive and presumed cured at 52 months.

Based upon their experience the authors correctly conclude that gamma detection alone was not sensitive and they have now moved on to performing both lymphoscintigraphy and intraoperative mapping.

This study points to some of the difficulties in establishing a new surgical technique in the setting of a rare disease with a relatively narrow "window of curability". First in their study and those from the Netherland's Cancer Institute (NCI) the patients with false negative findings often present later with incurable disease and die. Second, in the largest series reported the NCI group (using optimal technique) reported a false negative rate of $18 \%$ that is almost the same as that reported using physical examination. This has lead to subsequent technical modifications that could improve test performance but this is not guaranteed. Finally the learning curve is thought to be about 30 cases to gain proficiency (ref. 17 in the article) and thus is not practical for most urologists that are not in a referral setting.

Alternatively superficial inguinal lymphadenectomy detects all the first echelon lymph nodes at risk, is less morbid in contemporary reports, and in several reports was not associated with false negative findings (1-3). Thus I believe superficial inguinal lymphadenectomy in selected high risk patients to be the "standard" and Dynamic Sentinel Node Biopsy as reported here, by the NCI group, and in our own experience (ref. 3 below) to be a technique in evolution that is still experimental and requires further refinement in high volume centers.

\section{REFERENCES}

1. Bevan-Thomas R, Slaton JW, Pettaway CA: Contemporary morbidity from lymphadenectomy for penile squamous cell carcinoma: The M.D. Anderson Cancer Center experience. J Urol. 2002; 167: 163842.

2. Colberg JW, Andriole GL, Catalona WJ: Long-term follow-up of men undergoing modified inguinal lymphadenectomy for carcinoma of the penis. Brit J Urol. 1997; 79: 54-57.

3. Spiess PE, Bassett R, Izawa J, Kedar D, Busby JE, Wong F, et al.: Utility of pre-operative lymphoscintigraphy and intra-operative sentinel lymph node mapping in penile cancer: a different viewpoint. J Urol. 2006 (Suppl) 175: \#600.

Dr. Curtis A. Pettaway Professor of Urology The University of Texas M.D. Anderson Cancer Center Houston, Texas, USA E-mail: cpettawa@mdanderson.org 


\section{EDITORIAL COMMENT}

The presence of nodal metastases is the single most important factor in penile cancer. However, the timing of lymphadenectomy in patients presenting without clinical signs of node disease is still controversial. In the last years, there has been a growing awareness on the need of adopting better staging procedures in order to decrease the number of unnecessary node dissections and to improve detection of occult metastases.

The current European Association of Urology Guidelines recommend a risk-based approach for lymph node staging, including a wait and see strategy and surgical groin exploration using a low, intermediate and high risk stratification (1). In this setting, a new concept for lymph node staging, the so called dynamic sentinel biopsy (DSNB), has been introduced (2). Also in our experience, this technique offered similar results to those of radical lymphadenectomy but significantly lower morbidity (3).

Thus, the present report by Gonzaga-Silva et al. is interesting as it deals with a timely topic in urooncology. Of course, the major limitation of this prospective single institution study is the fact that the authors adopt an incomplete technique, as they clearly admit. This choice is questionable as they offer an already obsolete procedure to their 27 patients, with an expected limited clinical benefit. As a matter of fact, they recognize from the beginning that the real DSNB technique consists of different parts (i.e. preoperative lymphoscintigraphy, blue dye injection, intraoperative gamma ray detection), each one with a specific role in the reliability of the entire diagnostic procedure. They obviously found that the isolated gamma probe use has a very low sensibility and high false negative rate. Of note is that the authors also considered T1 tumors in their series, as recently suggested by Leijte et al. (4), as a risk of metastasis should be considered for these patients.

Finally, we agree with the authors when they state that DSNB procedure is difficult to master and it should be performed in centers with a minimum number of cases. We look forward to have the results from the same group of investigators after the introduction of the complete DSNB technique in their clinical practice as this should minimize false negative rates and optimize its diagnostic accuracy. In order to achieve this goal, a recent report by the group with the most extensive experience worldwide highlight some modifications to the original DSNB technique: fine needle aspiration cytology before lymphoscintigraphy; serial sectioning when analyzing the nodes; intraoperative palpation after injection of patent blue and surgical exploration in case of non-visualized groin (4). Further clinical research in this area is needed to better define the place of the sentinel node biopsy in the management of penile cancer patients. In this respect a very interesting approach has been proposed by TobiasMachado et al, who presented their encouraging results on 10 patients submitted to video endoscopic inguinal lymphadenectomy (5).

\section{REFERENCES}

1. Solsona E, Algaba F, Horenblas S, Pizzocaro G, Windahl T; European Association of Urology: EAU guidelines on penile cancer. Eur Urol. 2004; 46: 18.

2. Horenblas S, Jansen L, Meinhardt W, Hoefnagel CA, de Jong D, Nieweg OE, et al.: Detection of occult metastasis in squamous cell carcinoma of the penis using a dynamic sentinel node procedure. J Urol. 2000, 163: 100-4.

3. Perdona S, Autorino R, De Sio M, Di Lorenzo G, Gallo L, Damiano R, et al.: Dynamic sentinel biopsy in clinically node-negative penile cancer versus radical inguinal lymphadenectomy: a comparative study. Urology. 2005, 66: 1282-6.

4. Leijte JAP, Kroon BK, Valdes Olmos RA, Nieweg OE, Horenblas S: Reliability and safety of current dynamic sentinel node biopsy for penile carcinoma. Eur Urol 2007, in press. 


\section{Gamma Probe Technique for Sentinel Node Penile Carcinoma}

5. Tobias-Machado M, Tavares A, Ornellas AA, Molina WR Jr, Juliano RV, Wroclawski ER: Video endoscopic inguinal lymphadenectomy: a new minimally invasive procedure for radical management of inguinal nodes in patients with penile squamous cell carcinoma. J Urol. 2007, 177: 953-58.

Dr. Riccardo Autorino Clinica Urologica, AOU Policlinico Seconda Università Napoli, Italy

E-mail: ricautor@tin.it

Dr. Sisto Perdonà

UO Urologia, Istituto Nazionale Tumori

Fondazione G. Pascale Napoli, Italy 